\title{
A Simulation Research on Single Phase Bridge Full Control Resistive Load Rectifying Circuit Based on MATLAB
}

\author{
Han-hong $\operatorname{Tan}^{1}$, Xiang Zhao ${ }^{2}$ \\ 1. Guangdong University of Science \& Technology, Dongguan, Guangdong, 523083;2.Guilin \\ University of Electronic Technology,Guilin, Guangxi, 541004
}

\begin{abstract}
Keywords: Single Phrase Full Control Bridge; Resistive Load; Rectifying Circuit; Simulation Model Diagram
\end{abstract}

\begin{abstract}
The controlled rectifier circuit simulation study has been conducted in this paper with the MATLAB7.0 Simulink power system simulation model, the paper provides analysis waveform of a single phrase full control bridge resistive load circuit, provides the circuit's MATLAB simulation model diagram and the simulation waveform, and in this paper simulation waveforms and theory analysis waveforms were analyzed and compared. The results of simulation show that the model is correct. It is proved that the proposed model is convenient, flexible, convenient, intuitive, thus it provides an effective and auxiliary tool for the teaching of power electronics and experiments
\end{abstract}

\section{Introduction}

Rectifier circuit, especially single phase bridge controlled rectifier circuit, is the most important circuit in power electronics technology, and it is also the most widely applicable circuit, the circuit is not only used in the general industry, but it is widely used in other fields, including transportation, power system, communication system, energy system etc. It has practical significance to do comparative study on single phase bridge controlled rectifier circuit parameters, because it is not only a critical link in power electronic circuits theory learning, but has the predicting and guiding effect in the actual application of engineering practice. ${ }^{[1][2]}$ The visual simulation tool provided Matlab, Simulink, can establish the circuit simulation model directly, and it can change the parameters of the simulation at will, and the simulation result could be got immediately, and it has the feature of strong visuality, further eliminating the steps of programming. ${ }^{[3-5]}$ 。 As a new kind of high performance language, Matlab provides an ideal tool for the research and application of power electronic technology.

\section{Theoretical Analysis}

The single phrase full control bridge resistive load circuit principle diagram is shown in Figure 1:

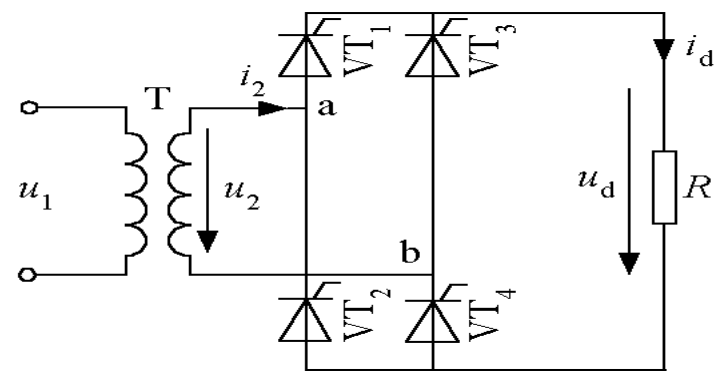

Figure1 The single phrase full control bridge resistive load circuit principle diagram

VT1 and VT4 constitute a pair of bridge arm, and it withstands voltage U2 in the U2 positive half cycle, and when there has the trigger pulse, it turns on, when there has the zero crossing U2, it turns off.

VT2 and VT3 constitute another pair of bridge arm, and it withstands voltage -u2 in the U2 
positive half cycle, and when there has the trigger pulse, it turns on, when there has the zero crossing U2, it turns off.

From the above theoretical analysis, the theoretical analysis waveform of the single phrase full control bridge resistive load circuit is shown in Figure 2. $u_{d}$ is the load $\mathrm{R}$ voltage and $i_{d}$ is the load current. $u_{V T 1,4}$ is the voltage of thyristor $V T 1$ and $V T 4, i_{2}$ is the current flowing through the transformer $\mathrm{T}$.

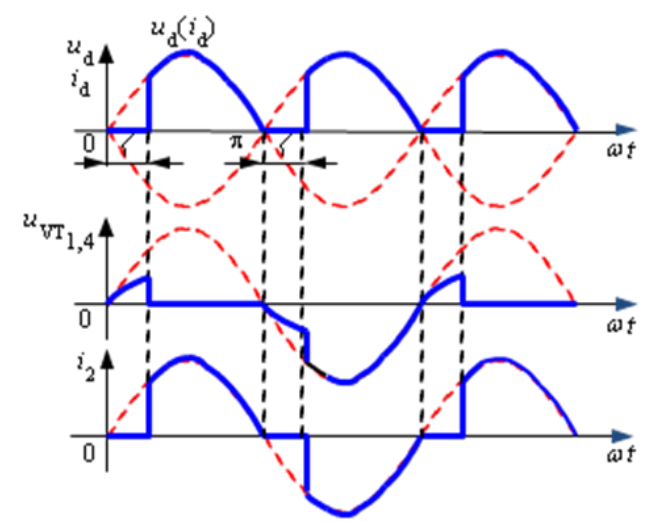

Figure 2 the theoretical analysis waveform of the single phrase full control bridge resistive load circuit

The theoretical analysis shows that the average value of the output DC voltage $u_{d}$ is:

$$
\begin{aligned}
& U_{\mathrm{d}}=\frac{1}{\pi} \int_{\alpha}^{\pi} \sqrt{2} U_{2} \sin \omega t \mathrm{~d}(\omega t)=\frac{2 \sqrt{2} U_{2}}{\pi} \frac{1+\cos \alpha}{2} \\
& =0.9 U_{2} \frac{1+\cos \alpha}{2}
\end{aligned}
$$

The average value of the output DC current $I_{d}$ is:

$$
I_{\mathrm{d}}=\frac{U_{\mathrm{d}}}{R}=\frac{2 \sqrt{2} U_{2}}{\pi R} \frac{1+\cos \alpha}{2}=0.9 \frac{U_{2}}{R} \frac{1+\cos \alpha}{2}
$$

\section{MATLAB Simulation Model}

The simulation model of single phase full wave rectifier circuit with resistive load of Simulink simulation module based on MATKAB7.0 is shown in Figure3.

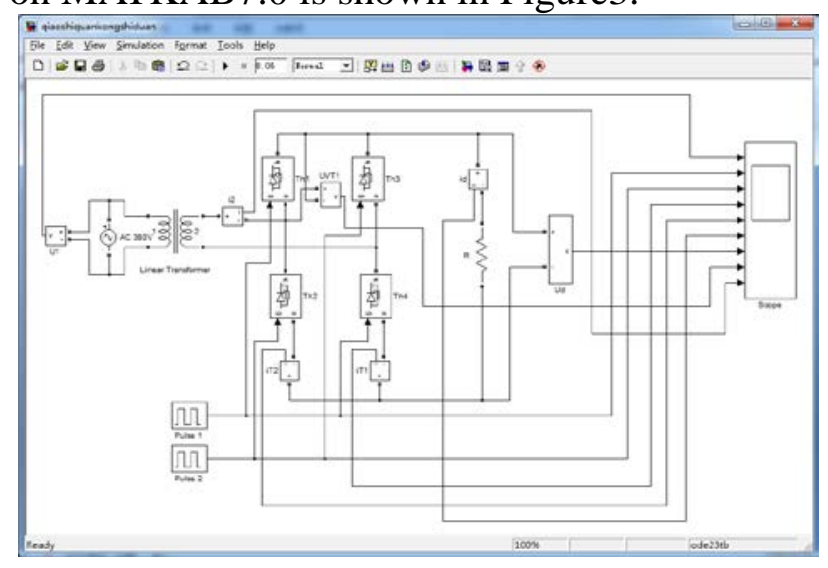

Figure 3 The simulation model of single phase full wave rectifier circuit with resistive load

The modules and extraction paths that are used in the simulation model are shown in Table 1. The parameter setting of Module $\mathrm{T}$ in the simulation model is shown in the Figure 4.

Table 1 


\begin{tabular}{|c|c|}
\hline Module Name & Extraction Path \\
\hline Pulse1、Pulse2 & Simulink\Source \Pulse Generator \\
\hline $\mathrm{T}$ & SimPowerSystems \Elements\Linear Transformer \\
\hline AC 380V & SimPowerSystems\Electrial Sources $\backslash$ AC Voltage Source \\
\hline Th1、Th2、Th3、Th4 & SimPowerSystems\Estra Library\Power Electronics $\backslash$ Thyristor \\
\hline U1、 Ud、UVT1 & SimPowerSystems \Measurements $\backslash$ Voltage Measurement \\
\hline iT1、iT2、id、i2 & SimPowerSystems \Measurements \Current Measurement \\
\hline $\mathrm{R}$ & SimPowerSystems\Elements\Parallel RLC Branch \\
\hline Scope & Simulink\Commonly Used Blocks\Scope \\
\hline & 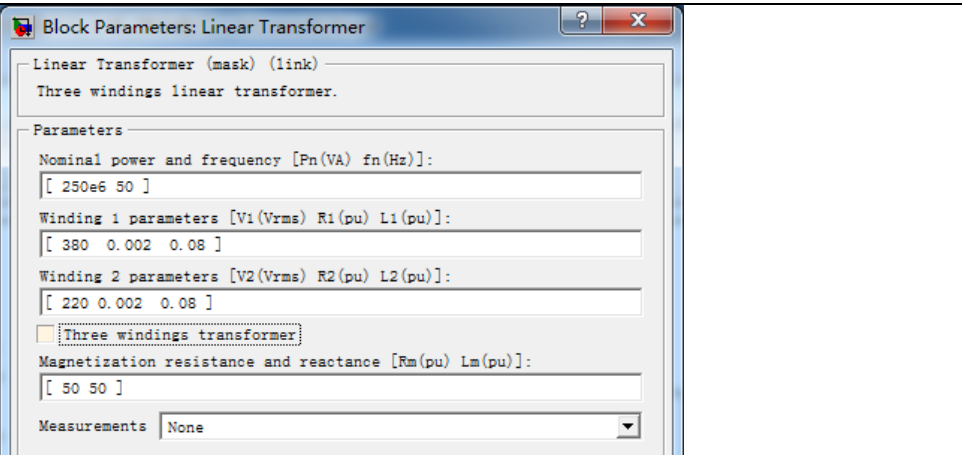 \\
\hline & 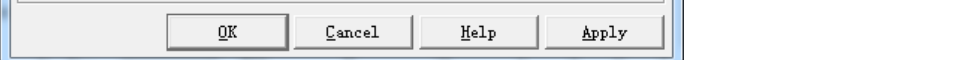 \\
\hline
\end{tabular}

Figure 4. The parameter setting of Module T

\section{Simulation Data and Wave Form}

In the MATLAB command window, when entering the following command, the output voltage of the rectifier circuit can be calculated.

>> syms U2 Ud alpha omega t;

$>>\mathrm{T}=1 / 50 ;$ omega $=100 *$ pi;

$>>\mathrm{u}=\operatorname{sqrt}(2) * \mathrm{U} 2 * \sin ($ omega $* \mathrm{t})$;

$>>\mathrm{Ud}=1 /($ pi/omega $) * \operatorname{int}(\mathrm{u}, \mathrm{t}, \mathrm{alpha} /$ omega,pi/omega);

$>>\mathrm{Ud} 1=\mathrm{vpa}(\mathrm{Ud}, 4)$

The command operation result is, $\mathrm{Ud} 1=.4500 * \mathrm{U} 2 *(1 .+\cos ($ alpha $))$

$U_{\mathbf{d}}=0.9 U_{2} \cos \frac{1+\alpha}{2}$ 。

The above analysis shows that the result of MATKAB command operation is consistent with the theoretical analysis.

According to the formula $t=\frac{\alpha T}{360^{\circ}}$, the grid alternating current is $\mathrm{T}=0.02 \mathrm{~s}$,. When the thyristor control angle $\quad \alpha=0^{\circ}, \mathrm{t}=0$ (while in the actual simulation model, the value of t should not be zero ( $t \neq 0$ ), and the $t$ should be $0.0001 \mathrm{~s}$ at this moment) ; when $\alpha=30^{\circ}, \mathrm{t}=0.00167 \mathrm{~s} ; \alpha=45^{\circ}$, $\mathrm{t}=0.0025 \mathrm{~s} ; \quad \alpha=60^{\circ}, \mathrm{t}=0.00333 \mathrm{~s} ; \quad \alpha=90^{\circ}, \mathrm{t}=0.005 \mathrm{~s}$, and so on.

When setting the parameters of the pulse signal generator, if $\alpha=60^{\circ}$, Pulse1's Phase Delay should be $0.00333 \mathrm{~s}$, Pulse2's Phase Delay should be $0.01+0.00333 \mathrm{~s}=0.01333 \mathrm{~s}$, because the interval 
of positive and negative half wave should be $0.02 \mathrm{~s}$.

The model 3 diagram has been simulated, the parameter of the model diagram is set, and the algorithmOde23tb or Oder15s has been selected. The wave form has been simulated from zero second to $0.06 \mathrm{~s}$. The simulation algorithm and simulation time parameter setting are shown in Figure 5. When the thyristor control angle $\alpha=60^{\circ}$, The simulation model of single phase full wave rectifier circuit with resistive load is shown in Figure 6, from top to bottom, they are $u_{1} 、 u_{g 1}$ 、 $u_{g 2} 、 i_{T 1} 、 i_{T 2} 、 i_{d} 、 u_{d} 、 u_{V T 1}$ and $i_{2}$ instantaneous waveform. $u_{1}$ is sinusoidal voltage instantaneous value, $u_{g 1}$ is the gate pulse of thyristor VT1 and VT4, $u_{g 2}$ is the gate pulse of thyristor VT2 and VT3, $i_{T 1}$ is the current flowing through thyristor VT1 and VT4, $i_{T 2}$ is the current flowing through thyristor VT2 and VT3, $i_{d}$ is load current, $\quad u_{d}$ is load voltage, $u_{V T 1}$ is the voltage of VT1, $i_{2}$ is the current flowing through the transformer $T . U_{d} 、 i_{d} 、 U_{V T 1}$ and $i_{2}$ 's simulation waveform enlarged drawing is shown in Figure7.

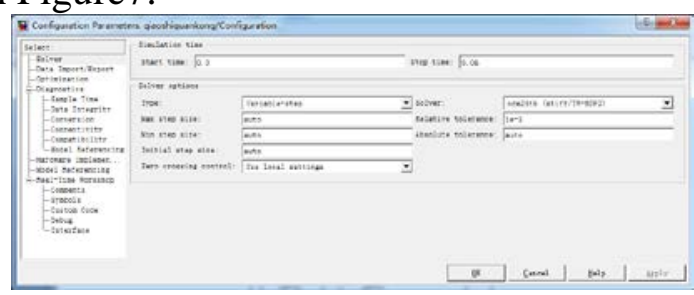

Figure 5 The simulation algorithm and simulation time parameter setting

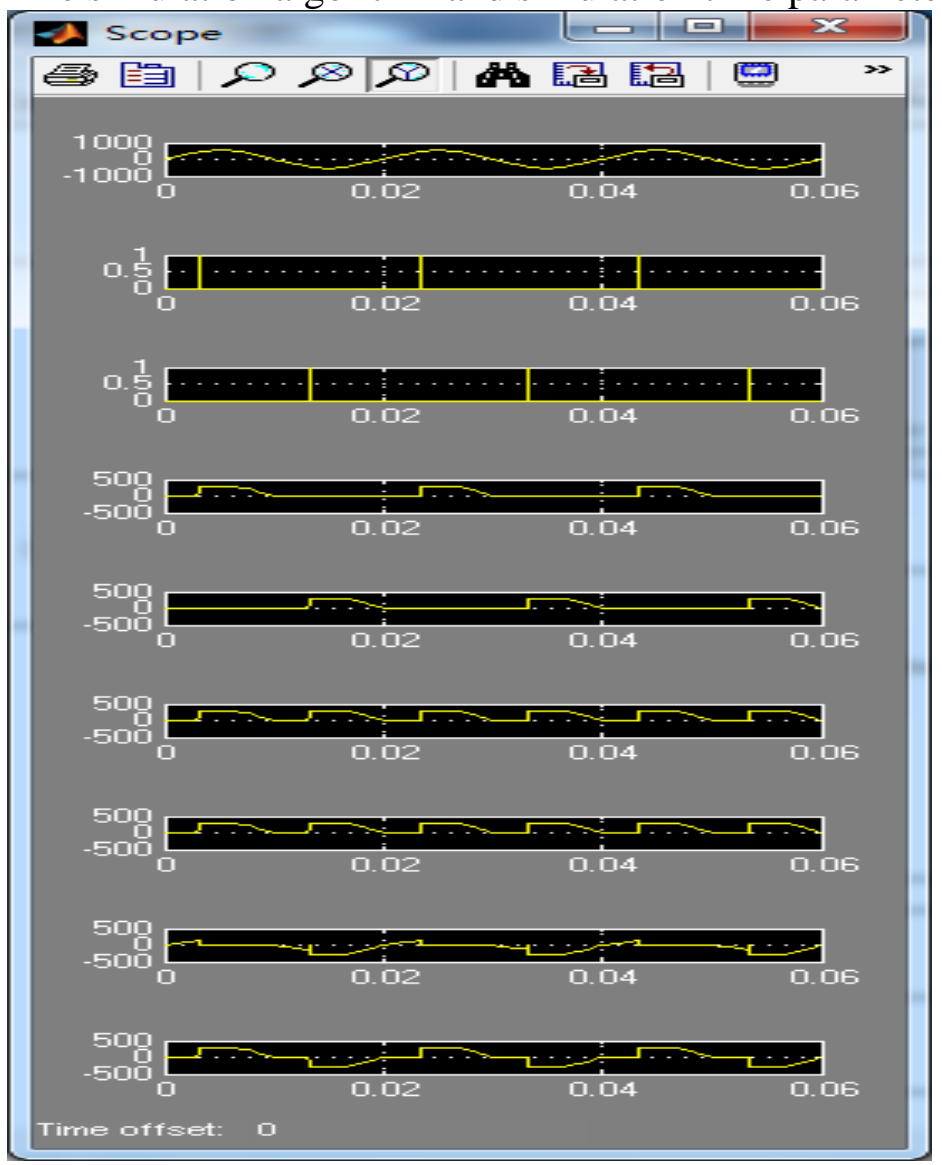

Figure $6 \alpha=60^{\circ}, u_{1} 、 u_{g 1} 、 u_{g 2} 、 i_{T 1} 、 i_{T 2} 、 i_{d} 、 u_{d} 、 u_{V T 1}$ and $i_{2}$ 's instantaneous waveform simulation waveform 


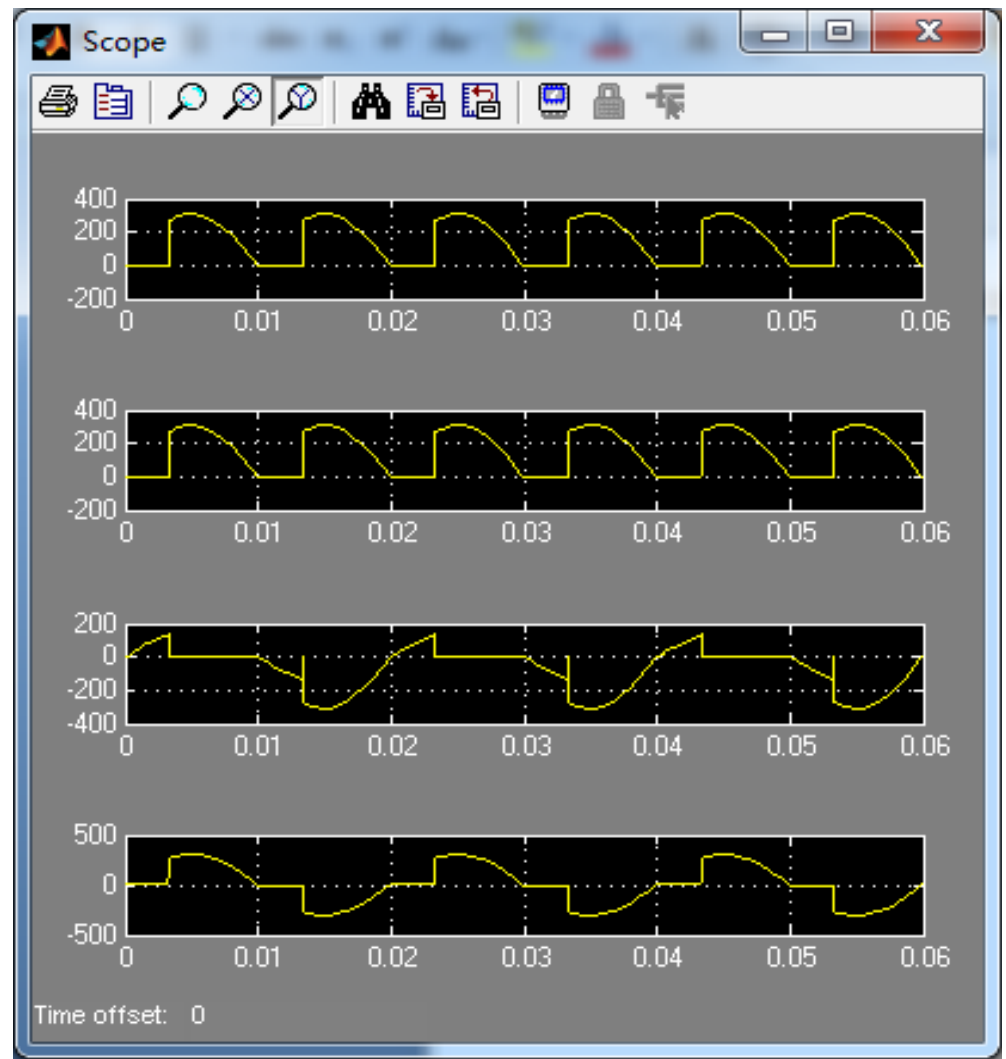

Figure $7 \alpha=60^{\circ}, \mathrm{U}_{\mathrm{d}} 、 i_{d} 、 \mathrm{U}_{\mathrm{VT} 1}$ and $i_{2}$ 's simulation waveform enlarged drawing

\section{Conclusion}

The MATLAB command operation results are consistent with the one of theoretical analysis. The theory and simulation results of single phase full wave rectifier circuit with resistive load have been analyzed with the application of Matlab visual simulation tool Simulink. The output voltage waveform that is obtained has been compared, and the paper further verifies the correctness of the simulation results. The paper validates the correctness of the model that has been built in this paper with the simulation analysis. Based on MATLAB/Simulink, single phase bridge rectifier circuit simulation analysis has been conducted in this paper, this method avoids the tedious drawing and calculation process in the conventional analysis methods, getting an intuitive and quick analysis method of rectifier circuit. The application of Matlab/ Simulink simulation can flexibly change the simulation parameters in the simulation process, and it can directly observe the simulation results that vary with parameters. The simulation research of rectifier circuit with application of Matlab lays the foundation of the analysis of single-phase bridge rectifier circuit and it is a powerful simulation software, which is worth popularizing and applying. It is also a good assistant tool for the experiment of power electronics technology.

\section{Foundation Project}

This paper is funded by the youth projects for creative talents in Guangdong province,Num(2014KQNCX244)

\section{References}

[1] Zhang Li. The Modern Power Electronics Technology Base [M]. Beijing. Higher Education Press. 1999.

[2] Jia Zhou. Wang Jinmei. Research on Single Phase Bridge Rectifier Circuit Based on MATLAB [J]. 2009 (6): 33-35,46. 
[3]Yan Xiaojun. Zhao Ni. Qin Hongjiang. etc. Design and Simulation of IIR Digital Filter Based on Matlab [J]. Computer and Modern, 2007 (6): 110-112.

[4]Pan Wenxia. Fan Yongwei. etc. Three Application Methods of Matlab in Circuit Teaching [J]. Power System And Its Automation, 2006, 18 (1): 108-112.

[5] WANG Jiu-he, YIN Hong-ren, ZHANG Jin-long, etc. Study on power decoupling control of three phase voltage source PWM rectifiers[C].[S.1.]: Power Electronics and Motion Control Conference, 2006.

[6] Ma Jun. Based on The Simulation of Matlab rectifier circuit [J]. Value Engineering, 2013,23 (82): 65-66.

[7]Hu Xuezhi. Modeling and Simulation of Three Phase Voltage Type PWM Rectifier Based on Matlab [J]. Automation and Instrumentation, 2005 (6): 73-75.

[8]Yang Yijin. Yin Huajie. Design of Voltage Type PWM Rectifier Controller [J]. Modern Electronic Technology, 2007, 30 (10): 169-172.

[9]Huang Kaizheng. Wang Xu. Research on the Improved Algorithm of Space Vector Control of PWM Rectifier [J]. applications, 2009, 28 (3): 52-54.

[10] Chen Aiwu. Liu Mouhui. Jiang Hui. Application of MATLAB/SIMUIINK in the Teaching of Power Electronics Technology Course. [J] Journal of Hunan University of Science and Engineering.2008,29 (8): 31- 34. 